\title{
Acute subarachnoid hemorrhage due to giant vertebrobasilar dolichoectasia
}

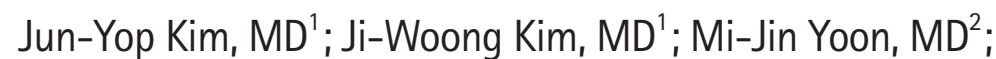
Seung-Han Suk, MD, PhD'

'Department of Neurology, Wonkwang University Sanbon Hospital, Wonkwang University School of Medicine, Gunpo, Republic of Korea

${ }^{2}$ Department of Radiology, Wonkwang University Sanbon Hospital, Wonkwang University School of Medicine, Gunpo, Republic of Korea

\section{Journal of Neurocritical Care \\ CASE REPORT \\ Received: May 6, 2019 \\ Revised: July 30, 2019 \\ Accepted: August 8, 2019 \\ Corresponding Author: \\ Seung-Han Suk, MD, PhD \\ Department of Neurology, Wonkwang University Sanbon Hospital, Wonkwang University School of Medicine, 327 \\ Sanbon-ro, Gunpo 15865, Republic of Korea \\ Tel: $+82-31-390-2300$ \\ Fax: +82-31-398-2223 \\ E-mail: suksh@wonkwang.ac.kr}

Background: Vertebrobasilar dolichoectasia (VBD) is a dilatative arteriopathy of the vertebrobasilar artery. Subarachnoid hemorrhage (SAH) is a rare but fatal complication of VBD.

Case Report: A 65-year-old man was brought to the emergency room with altered mental status. Computed tomography angiography revealed the presence of SAH, and the rupture site was suspected to be the dolichoectatic portion of the vertebrobasilar artery. Twelve years previously, the patient had developed a right pontine infarct, and VBD had been detected. The basilar artery had dilated to a diameter of $22 \mathrm{~mm}$ and contained a mural thrombus. The patient's noncompliance to antihypertensive treatment led to VBD progression and a hemifacial spasm. He was admitted to the intensive care unit for conservative treatment. Consciousness was restored, but he developed quadriplegia.

Conclusion: In patients with a giant VBD, strict blood-pressure control is important to prevent fatal complications. Targeted surgical or endovascular procedures may improve patient outcomes but require further studies.

Keywords: Vertebrobasilar dolichoectasia; Hypertension; Subarachnoid hemorrhage

\section{INTRODUCTION}

Vertebrobasilar dolichoectasia (VBD) is an arteriopathy resulting in pathological elongation, twisting, and dilatation of the vertebrobasilar artery. Dolichoectasia mostly occurs in the posterior cerebral circulation, but it can also occur in the anterior cerebral circulation; the basilar artery is involved in $>80 \%$ of cases. As the basilar artery gives off several branches at large angles, and the shearing forces are greater at the branching points, VBD can occur $[1,2]$.
Although parameters for the diagnosis of VBD have not been established, Smoke et al. [3] proposed certain diagnostic criteria based on information obtained from imaging studies, including radiography, angiography, and computed tomography (CT), of the vertebral arteries. VBD is diagnosed if the arterial diameter is $\geq 4.5 \mathrm{~mm}$ or if the separation or branching point of the dorsum sellae is above the suprasellar cistern (Table 1).

Here, we report a case of left arm paralysis caused by pontine hemorrhage in a patient who developed quadriplegia following

(C) 2019 The Korean Neurocritical Care Society

This is an Open Access article distributed under the terms of the Creative Commons Attribution Non-Commercial License (http://creativecommons.org/licenses/by-nc/4.0/) which permits unrestricted noncommercial use, distribution, and reproduction in any medium, provided the original work is properly cited. 
Table 1. Diagnostic criteria for basilar artery dolichoectasia based on computed tomography

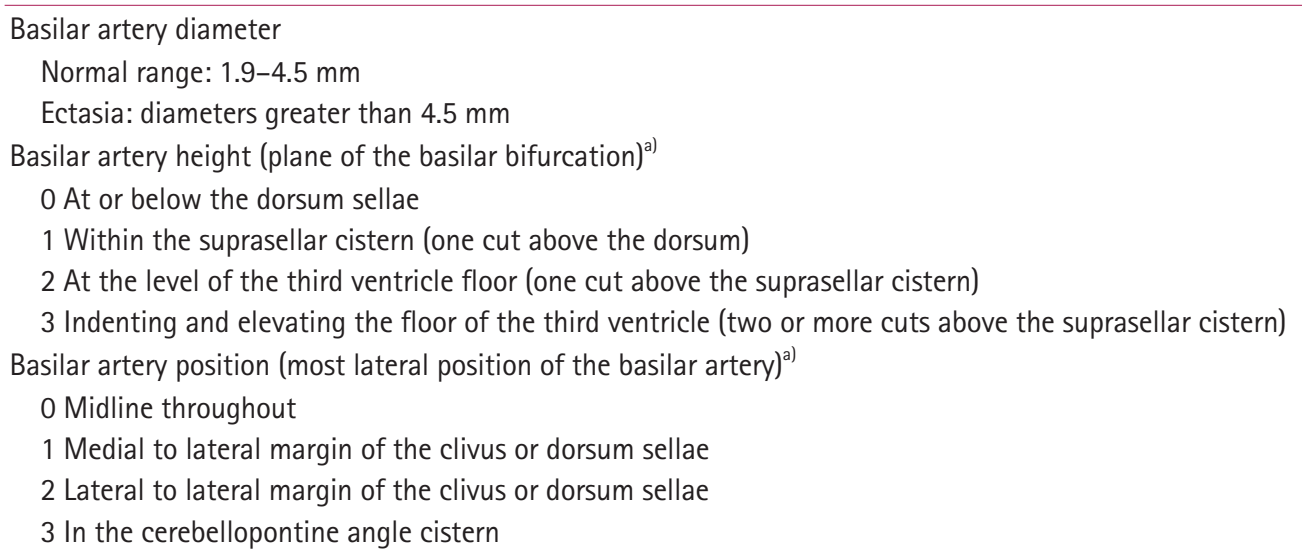

Modified from Smoker et al. AJNR Am J Neuroradio 1986;7:55-60, with permission of American Society of Neuroradiology [3].

a) Value of 2 or more suggests presence of abnormalities.

subarachnoid hemorrhage $(\mathrm{SAH})$ due to rupture of VBD.

\section{CASE REPORT}

In 2017, a 65-year-old man was brought to the emergency room with altered mental status. At the time of admission, his blood pressure was slightly elevated to $150 / 90 \mathrm{~mm} \mathrm{Hg}$, but the other vital signs were normal. Neurological examination showed that he was stuporous and unable to open his eyes on command. Limb weakness was not apparent, and he demonstrated an avoidance response to the painful stimulus. Both the pupils were $2 \mathrm{~mm}$ in diameter and showed normal light reactivity. Emergency CT angiography showed enlarged right vertebral and basilar arteries with a diameter of $23 \mathrm{~mm}$. The right vertebral artery twisted from the $\mathrm{V} 3$ portion to the V4 portion where it crossed to the left, and its diameter began to increase. The basilar artery enlarged until the point of origin of the superior cerebellar artery and compressed the left brainstem. Angiography revealed the presence of SAH, which was associated with intraventricular hemorrhage and hydrocephalus. SAH mainly involved the posterior fossa adjacent to the dilated basilar artery, near the basilar and sylvian cisterns, indicating that VBD was the rupture site (Fig. 1).

The patient had visited a hospital in 2002 with left upper limb motor weakness and dizziness and was diagnosed with a right pontine infarct. Magnetic resonance imaging of the brain revealed VBD with mural thrombus (Fig. 2). Therefore, the patient was treated with antiplatelet and antihypertensive agents to prevent both secondary stroke and complications of VBD. In the follow-up, a left hemifacial spasm was observed in 2003 because of a mass effect exerted by VBD. We considered intravascular surgery to control his symptoms, but because of the position and length of the dolichoectatic vertebrobasilar artery, the procedure would have carried a high risk, and the patient refused to undergo it. We continued the existing treatment and regularly monitored the patient. The patient was prescribed with medications and recommended necessary lifestyle modifications to control the blood pressure. However, because of noncompliance to the conservative therapy, his blood pressure was not controlled. In the follow-up visits, the doses of antihypertensive medications were continuously increased, as his blood pressure was uncontrolled. In 2014, his blood pressure was maintained at approximately 140/90 mm Hg even with four treatment regimens. We continued to emphasize on the need for increase in physical activity and lifestyle changes; however, he could not perform the suggested physical activities because of left upper limb muscle weakness. Moreover, he did not change his dietary behavior, and his body weight continued to increase. He developed diabetes in 2013. Five months before the onset of SAH, he was admitted to the hospital with sudden weight gain and hyperglycemia, which was uncontrolled with insulin. His blood pressure during hospitalization increased to $160 / 110 \mathrm{~mm} \mathrm{Hg}$, and he showed no response to various conservative treatments. This may have contributed to the development of SAH 15 years after the diagnosis of VBD.

The patient was admitted to the intensive care unit (ICU). We first administered intravenous nimodipine to control the blood pressure and prevent vasospasm. After admission, the patient had severe expectoration and developed fever. We continued antibiotic treatment for aspiration pneumonia. However, as the symptoms persisted and worsened, impeding the patient's breathing, we performed tracheostomy to maintain the airway. The patient developed quadriplegia on hospitalization day 5. On hospitalization day 7, he began to recover, with improvement in the level of consciousness. He could open his eyes on hospitalization day 14 . On 

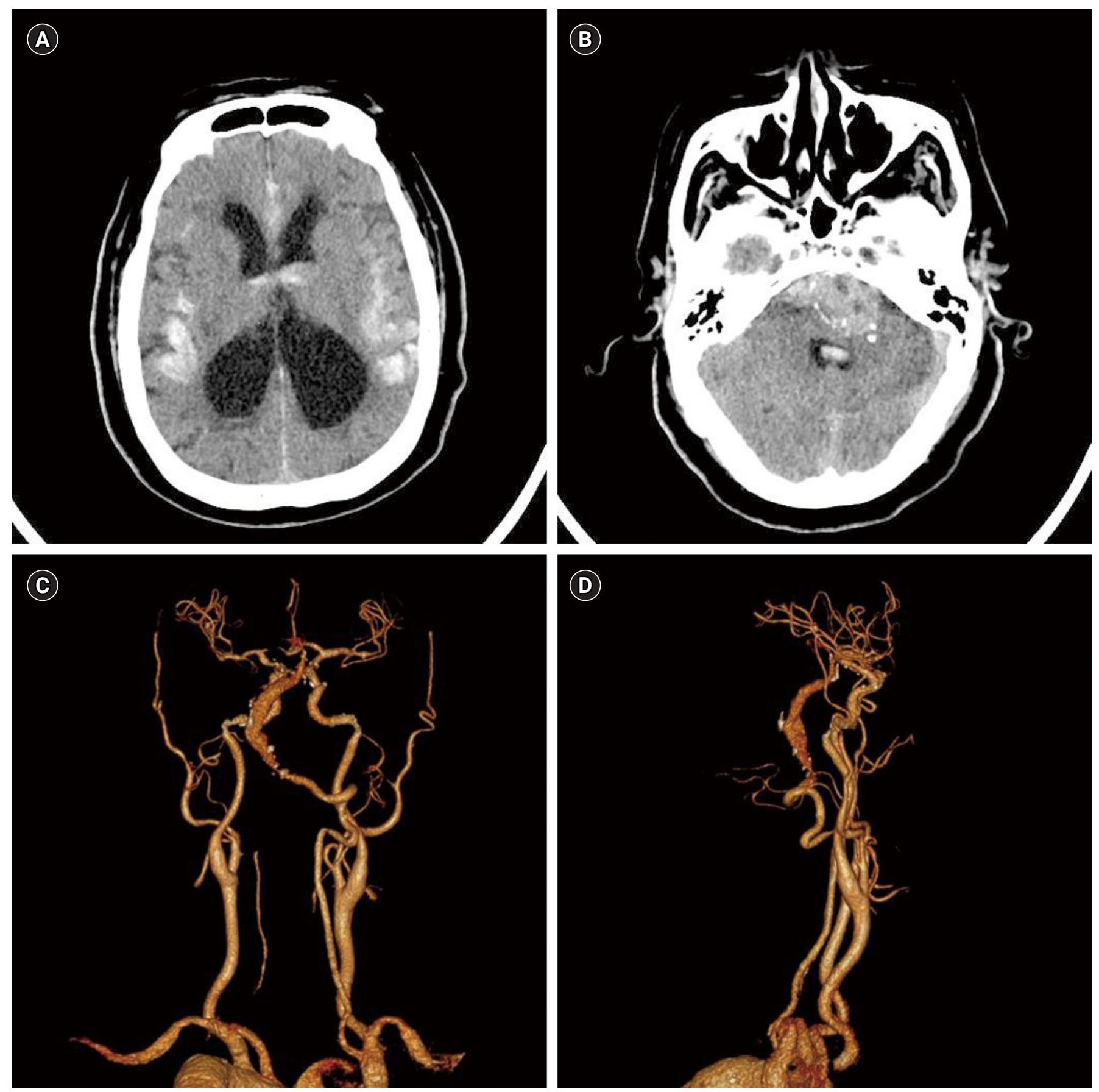

Fig. 1. (A) Computed tomography of the brain revealed subarachnoid hemorrhage with intraventricular hemorrhage and hydrocephalus. (B) Subarachnoid hemorrhage involved the posterior fossa near the fusiform aneurysm of the basilar artery, the basilar cistern, and both the sylvian cisterns. (C, D) Computed tomography angiography of the brain showing a giant vertebrobasilar dolichoectasia involving the right vestibular and basilar arteries.

hospitalization day 25 , the patient was completely conscious. Repeat CT showed partial resolution of subarachnoid and intraventricular hemorrhages. He was transferred to the general ward after approximately 30 days in ICU. He was recently admitted to a longterm care facility to manage his quadriplegic bedridden condition.

\section{DISCUSSION}

On histology, VBD shows fragmentation of the internal elastic lamina and hyperplasia of the intima. Neonangiogenesis progresses with thickened intima and is accompanied by intermural 

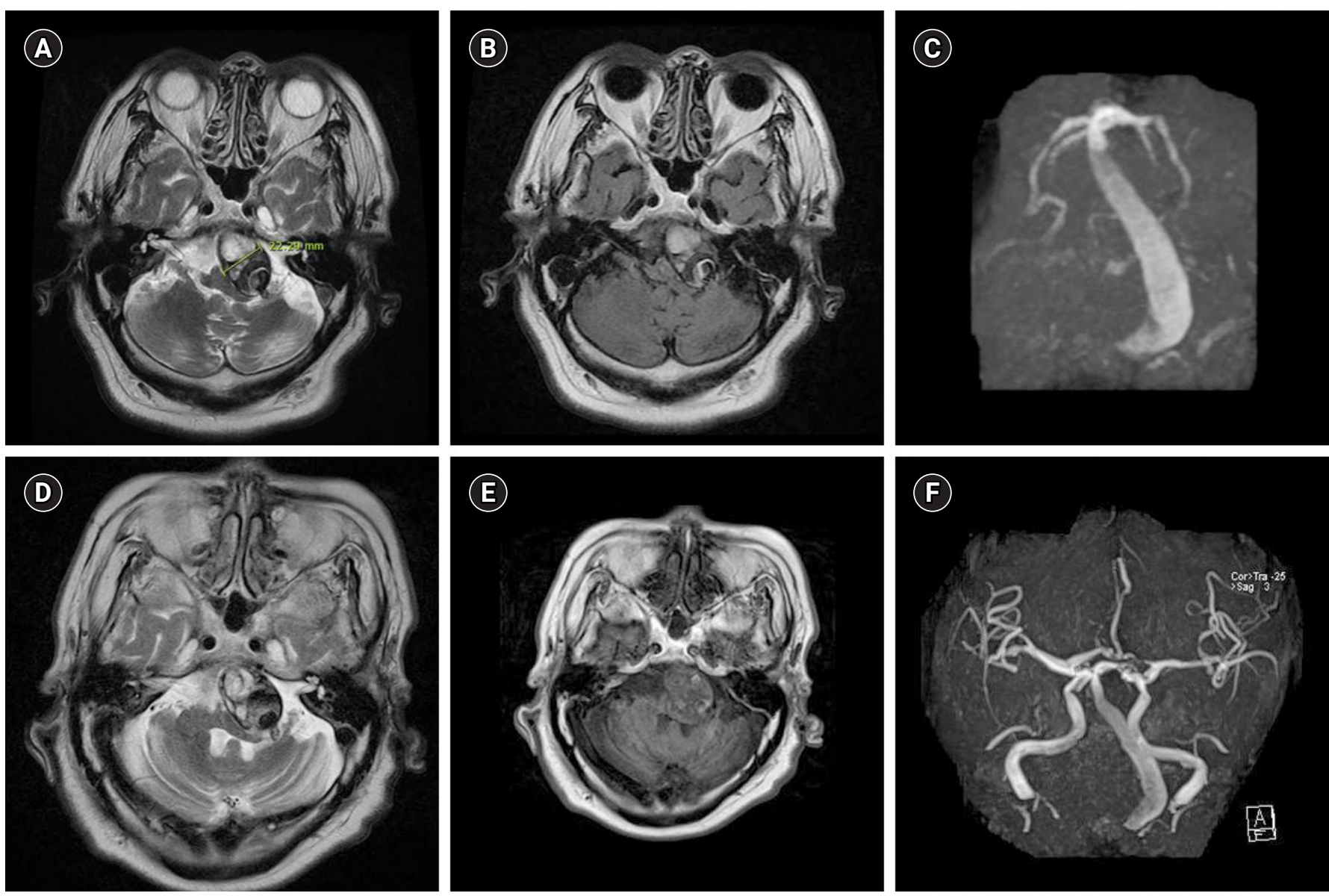

Fig. 2. ( $A, B)$ Magnetic resonance imaging (MRI) showing a giant vertebrobasilar dolichoectasia (22 mm in diameter) with a large mural thrombus within the tortuous basilar artery shifting the brainstem toward the right side. (C) Magnetic resonance angiography (MRA) demonstrating narrowing of the lumen of the basilar artery because of a thrombus. (D-F) MRI and MRA performed 5 months before the subarachnoid hemorrhage appeared showing no significant changes in the dimensions of the artery with vertebrobasilar dolichoectasia.

hemorrhage and thrombus [4].

Hemodynamic stress on the vessel wall causes degeneration of elastin and collagen, which comprise the internal elastic lamina, resulting in weakening of the arterial wall. Intimal thickening appears to adapt to these changes. Many risk factors are associated with these conditions. The age and sex of the patient, vascular causes, such as hypertension, metabolic disorders, such as Fabry and Pompe diseases, and hereditary diseases, such as Marfan syndrome, may affect the formation of vascular interstitial structures $[2,5]$.

Patients with VBD may be asymptomatic or present with symptoms of an ischemic cerebral infarction or transient cerebral ischemia due to thrombus formation. They may also experience intracranial hemorrhage due to rupture of the dilated vessel and become symptomatic because of compression of the cerebral nerves, brain stem, and ventricles [2].

To date, there is no known method to prevent VBD. Therefore, currently, empirical treatment is performed for secondary prevention and symptomatic control of the fatal complications of

\section{$\operatorname{VBD}[1,2]$.}

Intravascular thrombus formation is common in vessels with VBD, secondary to vasodilatation and decreased blood flow velocity. Therefore, occlusion of a perforated artery by a thrombus or embolus can occur with VBD [6]. If the ectatic vessels compress the cranial nerves, symptomatic manifestations, such as a hemifacial spasm and trigeminal neuralgia, may occur $[7,8]$. In addition, patients may present with dementia secondary to hydrocephalus, caused by compression of the third ventricle.[9]

$\mathrm{SAH}$ is observed in approximately $1 \%$ of the patients with VBD following rupture of the elongated and tortuous vessels. Although rare, SAH has a high mortality rate. Arterial rupture could be caused by local inflammation and ischemic changes in the blood vessel wall following a decrease in vascular resistance associated with the progress of vasodilation and thrombosis due to VBD [10].

The most common risk factors for VBD-related bleeding are the extent of venous dilatation and degeneration, arterial hypertension, the use of antiplatelet agents or anticoagulants, and the 
female sex [11]. To prevent rupture of VBD, strict blood pressure control is mandated. It is also necessary to exercise due caution while administering antiplatelet agents and anticoagulants for prevention of ischemic diseases in patients with a giant VBD [2].

Intravascular surgery can be considered to preempt the vessel rupture and reduce pressure on the nearby structures. However, as in our case, VBD in the basilar trunk may present with some clinical difficulties. Arteries with VBD do not have a defined neck. The basilar trunk has many perforating arteries supplying blood to the pons; surgery at this location is difficult [12].

Proximal ligation or parent vessel occlusion may be considered, but the overall prognosis is poor compared to dolichoectasia in the vertebral or posterior cerebral artery [13]. Recently, flow-diverting stents have been used in patients with VBD, but the overall prognosis is poor in patients with mass effects or SAH [14]. A study on the treatment options for these patients is needed.

In the present case, the patient was initially admitted and diagnosed with pontine infarction and started on medications. However, the patient did not adhere to the prescribed treatment or follow the recommended lifestyle changes to control hypertension. A hemifacial spasm occurred, but he rejected endovascular therapy.

In summary, comprehensive management of vascular risk factors, including strict control of blood pressure, is important for the prognostic outcome of a giant VBD. Endovascular therapy should be considered after weighing the risk and benefit to patients. Further studies and technical advances are needed.

\section{ARTICLE INFORMATION}

\section{Conflict of interest}

No potential conflict of interest relevant to this article.

\section{Funding}

This work was supported by the Wonkwang Research Grant in 2019.

\section{ORCID}

Jun-Yop Kim, https://orcid.org/0000-0002-4560-0291

Ji-Woong Kim, https://orcid.org/0000-0002-7432-2585

Mi-Jin Yoon, https://orcid.org/0000-0002-7799-3966

Seung-Han Suk, https://orcid.org/0000-0002-8732-5021

\section{Author contributions}

Conceptualization: JYK and SHS. Data curation \& Formal analysis: JYK and JWK. Visualization \& Writing-original draft:JYK, MJY, and SHS. Writing-review editing: JYK and SHS.

\section{REFERENCES}

1. Del Brutto VJ, Ortiz JG, Biller J. Intracranial arterial dolichoectasia. Front Neurol 2017;8:344.

2. Pico F, Labreuche J, Amarenco P. Pathophysiology, presentation, prognosis, and management of intracranial arterial dolichoectasia. Lancet Neurol 2015;14:833-45.

3. Smoker WR, Price MJ, Keyes WD, Corbett JJ, Gentry LR. High-resolution computed tomography of the basilar artery: 1 . normal size and position. AJNR Am J Neuroradiol 1986;7:5560.

4. Nakatomi H, Segawa H, Kurata A, Shiokawa Y, Nagata K, Kamiyama $\mathrm{H}$, et al. Clinicopathological study of intracranial fusiform and dolichoectatic aneurysms: insight on the mechanism of growth. Stroke 2000;31:896-900.

5. Mizutani T, Miki Y, Kojima H, Suzuki H. Proposed classification of nonatherosclerotic cerebral fusiform and dissecting aneurysms. Neurosurgery 1999;45:253-9.

6. Park JG, Kim BC, Lee SH, Choi SM, Park MS, Kim MK, et al. Brainstem infarction in patients with basilar artery dolichosis. J Korean Neurol Assoc 2005;23:318-23.

7. Rahman EA, Trobe JD, Gebarski SS. Hemifacial spasm caused by vertebral artery dolichoectasia. Am J Ophthalmol 2002; 133:854-6.

8. Ma X, Sun X, Yao J, Ni S, Gong J, Wang J, et al. Clinical analysis of trigeminal neuralgia caused by vertebrobasilar dolichoectasia. Neurosurg Rev 2013;36:573-7.

9. Siddiqui A, Chew NS, Miszkiel K. Vertebrobasilar dolichoectasia: a rare cause of obstructive hydrocephalus: case report. $\mathrm{Br} \mathrm{J}$ Radiol 2008;81:e123-6.

10. Sokolov AA, Husain S, Sztajzel R, Croquelois A, Lobrinus JA, Thaler D, et al. Fatal subarachnoid hemorrhage following ischemia in vertebrobasilar dolichoectasia. Medicine (Baltimore) 2016;95:e4020

11. Passero SG, Calchetti B, Bartalini S. Intracranial bleeding in patients with vertebrobasilar dolichoectasia. Stroke 2005;36: 1421-5.

12. Saliou G, Sacho RH, Power S, Kostynskyy A, Willinsky RA, Tymianski $\mathrm{M}$, et al. Natural history and management of basilar trunk artery aneurysms. Stroke 2015;46:948-53.

13. Coert BA, Chang SD, Do HM, Marks MP, Steinberg GK. Surgical and endovascular management of symptomatic posterior circulation fusiform aneurysms. J Neurosurg 2007;106:855-65.

14. Raphaeli G, Collignon L, De Witte O, Lubicz B. Endovascular treatment of posterior circulation fusiform aneurysms: single-center experience in 31 patients. Neurosurgery 2011;69: 274-83. 
